\title{
Large-Scale Network Coupling with the Fusiform Cortex Facilitates Future Social Motivation
}

\author{
Amanda V. Utevsky, ${ }^{1,2}$-David V. Smith, ${ }^{3}$ (D) Jacob S. Young, ${ }^{4}$ and ${ }^{-S c o t t ~ A . ~ H u e t t e l ~}{ }^{1,2}$ \\ DOI:http://dx.doi.org/10.1523/ENEURO.0084-17.2017 \\ ${ }^{1}$ Center for Cognitive Neuroscience, Duke University, Durham, NC 27708, ${ }^{2}$ Department of Psychology and \\ Neuroscience, Duke University, Durham, NC 27708, ${ }^{3}$ Department of Psychology, Temple University, Philadelphia, PA \\ 19122, and ${ }^{4}$ Pritzker School of Medicine, University of Chicago, Chicago, IL 60637
}

\begin{abstract}
Large-scale functional networks, as identified through the coordinated activity of spatially distributed brain regions, have become central objects of study in neuroscience because of their contributions to many processing domains. Yet, it remains unclear how these domain-general networks interact with focal brain regions to coordinate thought and action. Here, we investigated how the default-mode network (DMN) and executive control network (ECN), two networks associated with goal-directed behavior, shape task performance through their coupling with other cortical regions several seconds in advance of behavior. We measured these networks' connectivity during an adaptation of the monetary incentive delay (MID) response-time task in which human participants viewed social and nonsocial images (i.e., pictures of faces and landscapes, respectively) while brain activity was measured using fMRI. We found that participants displayed slower reaction times (RTs) subsequent to social trials relative to nonsocial trials. To examine the neural mechanisms driving this subsequent-RT effect, we integrated independent components analysis (ICA) and a network-based psychophysiological interaction (nPPI) analysis; this allowed us to investigate task-related changes in network coupling that preceded the observed trial-to-trial variation in RT. Strikingly, when subjects viewed social rewards, an area of the fusiform gyrus (FG) consistent with the functionally-defined fusiform face area (FFA) exhibited increased coupling with the ECN (relative to the DMN), and the relative magnitude of coupling tracked the slowing of RT on the following trial. These results demonstrate how large-scale, domain-general networks can interact with focal, domain-specific cortical regions to orchestrate subsequent behavior.
\end{abstract}

Key words: default-mode network; effective connectivity; executive control; faces; goal-directed behavior

\section{Significance Statement}

A diverse set of behaviors, from normal to pathologic, has been linked to the responses of large-scale functional networks. Yet, it remains unclear how these domain-general networks shape subsequent processing in cortical regions with domain-specific function. Here, we examine how two such networks, the default-mode network $(\mathrm{DMN})$ and executive control network (ECN), connect functionally with other cortical regions to alter performance in an incentive-compatible task. We found that differential coupling between a prototypical face processing region and DMN and ECN tracked subsequent improvements in performance to social stimuli. Our approach allowed us to examine direct coupling with functional networks to future behavior, providing a significant step forward in understanding how large-scale networks coordinate thought and action.

\section{Introduction}

Since the discovery of functionally correlated brain regions (Biswal et al., 1995), large-scale functional networks

Received March 15, 2017; accepted September 26, 2017; First published October 3, 2017

The authors declare no competing financial interests. have been considered fundamental features of brain activity (Gusnard and Raichle, 2001; Beckmann et al., 2005; Behrens and Sporns, 2012). These networks are highly

Author contributions: A.V.U., D.V.S., J.S.Y., and S.A.H. designed research; A.V.U., D.V.S., and J.S.Y. performed research; A.V.U., D.V.S., and J.Y.S. analyzed data; A.V.U., D.V.S., and S.A.H. wrote the paper. 
reliable across large samples of participants (Biswal et al., 2010; Smith et al., 2013) and are thought to reflect intrinsic properties of brain organization. Many of these networks reflect sensory and perceptual processes instantiated within visual or auditory regions (Smith et al., 2009). Still others contribute broadly to cognitive processing across stimulus and task domains, including the default-mode (DMN), executive control (ECN), and frontoparietal networks (Dosenbach et al., 2007; Smith et al., 2009). These functional networks have been linked to various aspects of behavior including demographic variables (Filippini et al., 2009; Cowdrey et al., 2014), traits (Shannon et al., 2011; Kucyi et al., 2014), and cognitive states (Smith et al., 2009; De Havas et al., 2012).

Yet, for effective behavior in a particular task, these large-scale domain-general networks must alter ongoing task-specific processing in focal brain regions. To claim that such interactions (i.e., between large-scale networks and specific brain regions) are critical for behavior change, several conditions should be met. First, a largescale network should be identifiable during task performance independently of other concurrent activation; that is, networks should be able to be extracted regardless of other processes occurring in focal brain regions that might contribute to that task (i.e., without relying on seedbased analyses; Cole et al., 2010; Smith et al., 2014b). Second, the coupling between a given network and a given focal brain region should systematically vary across task conditions according to the relative engagement of the task (Friston et al., 1997; O'Reilly et al., 2012). Third, those changes in coupling (e.g., effective connectivity; Friston, 2011) should predict the characteristics of subsequent behavior, to provide evidence that the coupling contributes to effective task performance. If these conditions are met, there would be strong evidence that coupling between a functional network and a focal brain region contributes to a specific cognitive process.

In the current study, we examined effective connectivity between large-scale networks and focal brain regions while subjects played a reaction times (RTs) game to receive social and nonsocial rewards in a modified monetary incentive delay (MID) task (Knutson et al., 2000). We have previously used this task to produce meaningful variability in reaction time behavior that reflects relative motivation (Clithero et al., 2011). We focused on the DMN and ECN since both have been linked to task performance, engagement, and other markers of executive function

and

This work was supported by the National Institutes of Health Grant NIMH RC1-88680, an Incubator Award from the Duke Institute for Brain Sciences (S.A.H.), and by National Institute of Mental Health National Research Service Awards F31-MH105137 (to A.V.U.) and F32-MH107175 (to D.V.S.)

Acknowledgments: We thank Christopher Coutlee and Cary Politzer for their assistance in task design and data collection.

Correspondence should be addressed to Scott A. Huettel, Box 90999, Duke University, Durham, NC 27708, E-mail: scott.huettel@duke.edu.

DOI:http://dx.doi.org/10.1523/ENEURO.0084-17.2017

Copyright (C) 2017 Utevsky et al.

This is an open-access article distributed under the terms of the Creative Commons Attribution 4.0 International license, which permits unrestricted use, distribution and reproduction in any medium provided that the original work is properly attributed. preparatory behavior. Behaviorally, we found that participants exhibited slower response times (RTs) subsequent to social relative to nonsocial trials, reflecting a change in motivation according to social stimulus type. We investigated whether coupling with DMN and ECN contributed to this subsequent-RT effect by combining independent components (ICA) and psychophysiological interactions (PPIs) analyses. This network-based PPI (nPPI) pipeline allowed us to examine how DMN and ECN contribute to other cortical regions to shape subsequent motivated behavior up to several seconds later. Strikingly, we found that $\mathrm{DMN}$ and $\mathrm{ECN}$ differentially coupled with a region in the fusiform gyrus (FG) when subjects viewed the social rewards, and that changes in this coupling tracked the effect of stimulus type on subsequent RT. This region of the $F G$ is consistent with the functionally-defined fusiform face area (FFA), a region classically associated with face processing (Kanwisher et al., 1997; McCarthy et al., 1997).

These findings highlight functional network interactions that guide subsequent changes in reaction time behavior. While the cognitive mechanisms underlying the behavioral effect of previous stimulus type on subsequent reaction time require future research, we propose that this effect may be driven by increased attentional interference of social rewards relative to nonsocial rewards or a subsequent change in task motivation. Collectively, these results indicate that functional networks associated with goal-directed behavior can interact with focal brain regions to support future motivated behavior.

\section{Materials and Methods}

\section{Participants}

A group of 50 self-reported heterosexual males completed the task (mean age: 23.8 years, range 18-32); this sample size was established before data collection. All participants were screened before data collection to rule out prior or current psychiatric or neurologic illness. We excluded four participants because of data quality issues (see below, Preprocessing), and we excluded five participants because of a malfunctioning button box in the scanner. These exclusions left a final sample of 41 participants (mean age: 24.1 years, range: 18-32). All participants gave written informed consent as part of a protocol approved by the Institutional Review Board of Duke University Medical Center.

\section{Stimuli and tasks}

The experiment consisted of four components: (1) a training session outside the scanner; (2) a modified MID task (Knutson et al., 2000, 2001); (3) a passive viewing task with results previously published (Young et al., 2015); and (4) two additional postscan rating tasks. All participants received variable cash payment depending on their performance.

In advance of the neuroimaging session, participants memorized the associations of four fractal images with different potential rewards (example in Fig. 1). On the day of the scan, subjects were tested on the fractal images' associations to ensure they remembered the meaning of each image; if participants identified every association 


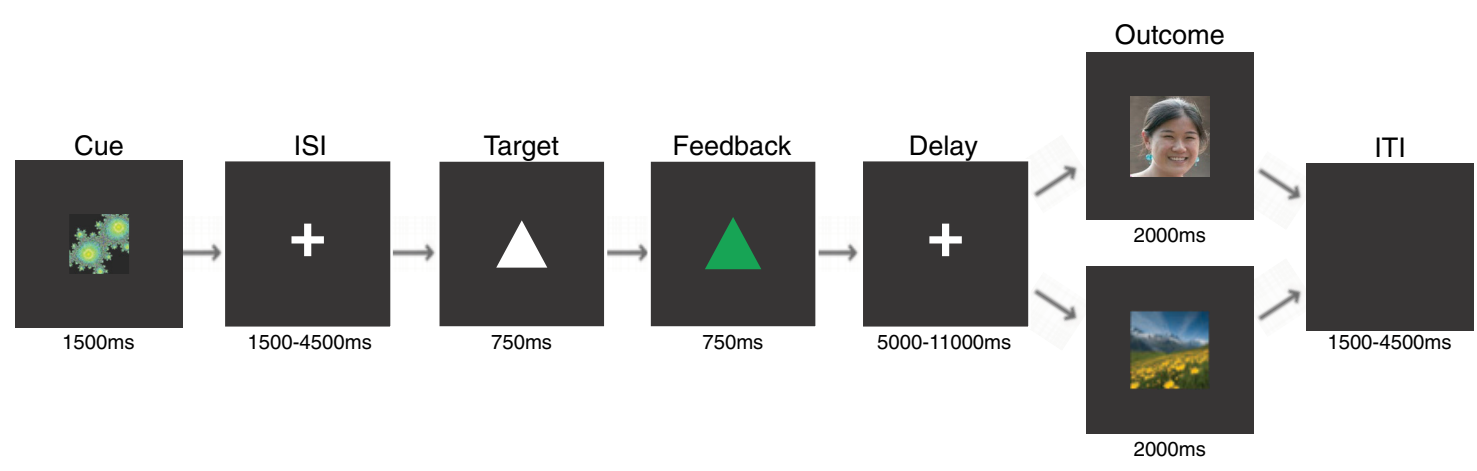

Figure 1. Task design. Fifty heterosexual men performed a modified MID task which examined timed responses to view social and nonsocial images as rewards (faces and landscapes, respectively). Each trial of the task began with an abstract image presented as a cue for $1500 \mathrm{~ms}$ indicating the potential reward for that trial. Following the cue, a fixation cross appeared for a variable duration (interstimulus interval, $1500-4500 \mathrm{~ms}$ ), followed by a target (a white triangle) that appeared on the screen for $750 \mathrm{~ms}$; participants responded by pressing a button box with their right index finger before the target disappeared. After the target, a feedback image (a colored triangle) appeared for $750 \mathrm{~ms}$; the feedback image was colored green if the participants' response was sufficiently fast on the trial (a win), and was blue if the response was too slow (a loss). After the feedback image disappeared, the participants waited a delay period that varied according to the cue and whether the subject won or lost that trial. Lastly, the reward image (either a face or a landscape) was shown for $2000 \mathrm{~ms}$. Consent was provided for the use of the image in this figure.

correctly on the first attempt $(n=31)$, they received an extra $\$ 5$ in addition to their study compensation. All participants successfully learned the image associations before entering the scanner.

Each trial of the modified MID task began with a fractal image presented as a cue for $1500 \mathrm{~ms}$ (Fig. 1). The different cues indicated the different potential benefits of a fast response on that trial: (1) reducing the delay until the presentation of a face image of a variable attractiveness rating (social delay; SD); (2) increasing the attractiveness of a face image presented after a variable duration (social attractiveness; $\mathrm{SA}$ ); (3) reducing the delay until the presentation of a landscape image of a variable attractiveness rating (nonsocial delay; ND); and (4) increasing the attractiveness of a landscape image presented after a variable duration (nonsocial attractiveness; NA).

Following the cue, a fixation cross appeared for a variable duration (interstimulus interval; $1500-4500 \mathrm{~ms}$ ), followed by a target (a white triangle) that appeared on the screen for $750 \mathrm{~ms}$. Participants then responded by pressing a button box with their right index finger before the target disappeared. A feedback shape then appeared for $750 \mathrm{~ms}$; that shape was colored green if the participant's response was sufficiently fast (a win), and was colored blue if the response was too slow (a loss). After the feedback image disappeared, participants waited a delay period that varied according to the cue and whether the subject won or lost that trial. Lastly, the reward image (either a face or a landscape) was shown for $2000 \mathrm{~ms}$, and there was an intertrial interval (ITI) that was jittered between 1500 and 4500 ms (with the 2000-ms reward image duration, the minimum and maximum time delays between reward onset and subsequent cue onset was 3500 and $6500 \mathrm{~ms}$, respectively).

Each run of the modified MID task consisted of 36 trials, nine images of each SD, SA, ND, and NA in randomized order. Across all runs included in the analyses, the four types of transitions (e.g., social followed by social, social followed by nonsocial, etc.) each occurred on $\sim 25 \pm 1 \%$ of trials, reflecting effective randomization of stimulus types across trials. Social images were photographs of young adult women and were cropped to show only the face (images were drawn from Smith et al., 2010). Nonsocial images were photographs of landscapes, and did not contain any body or facial features. All images were resized to uniform dimensions. Attractiveness (high/medium/low) of images was determined by ratings by an independent group. Additionally, performance thresholds were determined independently for each subject by an algorithm that adapted the task difficulty so that accuracy would be $\sim 60 \%$ (observed hit rate $=60.7 \%$ ).

After completing the four runs of the modified MID task, participants completed an unrelated passive-viewing task described elsewhere (Young et al., 2015). Following this second scanner task, participants completed two tasks outside the scanner. In the first task, participants rated the attractiveness of each image they viewed in the modified MID task on a scale of 1-7. Each participant's highestrated face and landscape images were then used in a second task involving forced choices between a landscape image and a face image presented simultaneously.

All tasks were programmed and displayed using the Psychophysics Toolbox (version 3; Brainard, 1997) for MATLAB (Mathworks).

\section{Behavioral analysis}

Behavioral data were analyzed using MATLAB and IBM SPSS Statistics 20. A RT difference was calculated for each participant by subtracting their mean RT on the trials subsequent to nonsocial trials from their mean RT on trials subsequent to social trials. Consequently, positive RT differences indicate shorter RTs subsequent to nonsocial trials (relative to social trials), whereas negative differences indicate shorter RTs subsequent to social trials (relative to nonsocial trials).

To control for factors other than prior stimulus category, such as attractiveness of the prior trial's reward image, the prior trial's RT, and the subsequent trial's stimulus 
type, we incorporated these factors into a general linear model (GLM) for each subject. The GLM included the following as regressors: (1) stimulus type on previous $(t-1)$ trial; (2) RT on previous trial; (3) attractiveness rating of the reward image of the previous trial; and (4) stimulus type on current trial $t$. This analysis indicates the specific influence of each regressor on the current trial's RT, and allowed us to examine the unique influence of the previous trial's stimulus category (social or nonsocial) on current RT. In our analyses, positive stimulus type $\beta$ weights reflect slower RTs on or following social trials, whereas negative stimulus type $\beta$ weights reflect faster RTs on or following social trials.

\section{Image acquisition}

Neuroimaging data were collected using a General Electric MR750 3.0 Tesla scanner equipped with an 8-channel parallel imaging system. We used a $\mathrm{T}_{2}$ *weighted spiral-in sensitivity encoding sequence (SENSE factor $=2$ ), with slices parallel to the axial plane connecting the anterior and posterior commissures [repetition time (TR): $1580 \mathrm{~ms}$; echo time (TE): $30 \mathrm{~ms}$; matrix: $64 \times$ 64; field of view (FOV): $243 \mathrm{~mm}$; voxel size: $3.8 \times 3.8 \times$ $3.8 \mathrm{~mm}$; 37 axial slices; flip angle: 70 degrees]. The first eight volumes of each run were removed to allow for magnetic stabilization. We additionally acquired wholebrain high-resolution anatomic scans (T1-weighted FSPGR sequence; TR: $7.58 \mathrm{~ms}$; TE: $2.93 \mathrm{~ms}$; matrix: $256 \times 256$; FOV: $256 \mathrm{~mm}$; voxel size: $1 \times 1 \times 1 \mathrm{~mm}$; 206 axial slices; flip angle: 12 degrees) to allow for coregistration and normalization.

\section{Preprocessing}

Our preprocessing used tools from the FMRIB Software Library package (FSL version 4.1.8; http://www.fmrib.ox. ac.uk/fsl/; Smith et al., 2004; Woolrich et al., 2009). We corrected for head motion by realigning the time series to the middle volume (Jenkinson and Smith, 2001), and then removed nonbrain material using a brain extraction tool (Smith, 2002). We then corrected intravolume slice-timing differences using Fourier-space phase shifting to align to the middle slice (Sladky et al., 2011). After spatially smoothing the image using a 5-mm full-width-halfmaximum isotropic Gaussian kernel, we applied a highpass temporal filter with a 100-s cutoff, and we normalized each 4-dimensional dataset to the grandmean intensity using a single multiplicative factor. Lastly, we spatially normalized the functional data to the Montreal Neurologic Institute (MNI) Template avg152 T1weighted template (3-mm isotropic resolution) using a 12-parameter affine transformation implemented in FLIRT (Jenkinson and Smith, 2001).

As part of our preprocessing and quality control, we additionally examined three partially correlated measures of quality assurance: signal-to-fluctuation-noise ratio (SFNR; Friedman and Glover, 2006), volume-to-volume head motion, and number of motion spikes within the time series (motion spikes were identified by evaluating the root-mean-square-error of each volume relative to the middle time point). Measures on each metric were considered outliers if they exceeded the 75 th percentile plus the value of $150 \%$ of the interquartile range (i.e., a standard boxplot threshold); runs that were identified as outliers were excluded from further analyses. Additionally, any participant who had fewer than two good runs (out of four total runs) was excluded from further analyses. These criteria eliminated four participants.

\section{Neuroimaging analysis}

To best meet the conditions for identifying task-specific interactions between large-scale networks and focal brain regions (see Introduction), our neuroimaging analyses proceeded in two phases, each described in a separate section below. First, we used ICA (Beckmann and Smith, 2004) and spatial regression (Filippini et al., 2009) to identify the large-scale neural networks of interests (DMN and ECN) and to examine the networks' levels of activation over the course of each run. Second, we used generalized nPPI models (adapted from McLaren et al., 2012) to identify brain regions whose coupling with the ECN and DMN changed as a function of the effect of stimulus type on subsequent RT. Importantly, this ICA-based nPPI approach follows the logic of region of interest (ROI)-based PPI analyses, with the critical difference of examining connectivity with data-driven large-scale neural networks instead of a specific seed region. Critically, we note that this nPPI pipeline allowed us to test the three necessary conditions for inferences that functional networks alter task-specific processing in focal brain regions.

\section{Identifying large-scale functional networks}

We used FSL's Multivariate Exploratory Linear Decomposition into Independent Components (MELODIC) version 3.10 to identify large-scale functional networks in the neuroimaging data (Beckmann and Smith, 2004). MELODIC ICA was implemented using temporal concatenation, which looks for common spatial patterns of components across participants' data without assuming a specific or common time course across all participants; we note that previous research has also used temporal concatenation-based ICA on task-based fMRI data (Utevsky et al., 2014; Young et al., 2015). The preprocessed data were whitened and projected into a 25-dimensional subspace (Ray et al., 2013; Utevsky et al., 2014). The whitened data were decomposed into sets of vectors describing the temporal and spatial signal variation, using a fixed-point iteration technique to optimize nonGaussian spatial source distribution (Hyvärinen, 1999). The estimated component maps were then thresholded by dividing the maps by the standard deviation of the residual noise, then fitting a Gaussian- $\gamma$ mixture model to the histogram of the normalized intensity values (Beckmann and Smith, 2004). This first step provided a datadriven means to identify functional networks present during task performance; this allowed us to meet the first condition for identifying task-specific interactions between large-scale networks and specific brain regions.

All unthresholded spatial maps from the ICA were then submitted to a spatial regression (part of FSL's dual regression analysis) to estimate the time courses of each network (Filippini et al., 2009; Leech et al., 2011). In this analysis, spatial maps are regressed onto each partici- 
pant's functional data, resulting in a matrix of $\mathrm{T}$ (time points) $\times \mathrm{C}$ (components) $\beta$ coefficients that characterize each subject's time courses for each network.

\section{Characterizing reward-related network connectivity and activation}

We assessed task-dependent network coupling using a generalized nPPI model. The generalized PPI, in contrast to a standard PPI, computes a separate PPI term for each task condition. This approach has been shown to yield more accurate estimates of how connectivity varies as a function of psychological context (McLaren et al., 2012). The generalized nPPI analysis was conducted using FMRI Expert Analysis Tool (FEAT) version 5.0.1.

The run-level model included six task regressors: social cue (duration $=1.5-4.5 \mathrm{~s}$ ), nonsocial cue (duration = 1.5-4.5 s), hits (duration $=0.75 \mathrm{~s}$ ), misses (duration = $0.75 \mathrm{~s}$ ), social reward outcome (duration $=2 \mathrm{~s}$ ), and nonsocial reward outcome (duration $=2 \mathrm{~s}$ ). We additionally included time courses of both the DMN and ECN that were produced by the spatial regression. Because of the minimum ITI of 1500 ms and the potential confound of the cue presentation occurring between the outcome and subsequent target phases of the task, we examined whether there was any collinearity between the outcomes and cues across the runs included in our analysis. For each run included in our analyses, we calculated the correlation values between the face and land outcome regressors and the face and land cue regressors, and then averaged these correlation values across all runs. The mean correlation values between face or land cues and face or land outcomes ranged from $r=-0.25$ to $r=$ -0.17 (minimum $r=-0.28$; maximum $r=-0.10$ ), indicating that our run-level analyses were able to isolate and model cues and outcomes independently, with minimal collinearity between regressors.

For our network interaction analysis, nPPI regressors were formed by multiplying the DMN and ECN time courses (zeroed to the mean of the time course), separately, by the social outcome and nonsocial outcome regressors (zeroed to the minimum value of the task time course; McLaren et al., 2012); this yielded four nPPI regressors: (1) DMN*social; (2) DMN*nonsocial; (3) ECN*social; and (4) ECN*nonsocial. To control for motion in the scanner, we additionally included motion spikes and motion parameters as regressors. Lastly, to control for the influence of other networks and potential artifacts on our generalized $\mathrm{nPPI}$, we included the time courses of the remaining 23 components from the ICA. The $\mathrm{nPPI}$ analysis allowed us to examine task-specific coupling between ECN and DMN with other regions in the brain, fulfilling the second condition for identifying the task-specific interactions of interest.

Subject-level analyses for the generalized nPPI were run using FEAT and implementing FMRIB's Local Analysis of Mixed Effects (FLAME 1), and examined activation across runs within each participant. Group-level analyses included each subject's demeaned $\beta$ s from the prior stimulus-category regressor (see above, Behavioral analysis); this allowed us to examine whether network cou- pling predicts the characteristics of subsequent RT, and fulfill the third condition for identifying task-specific interactions of interest. The group-level analysis additionally included the main effect of group and three motionrelated parameters (SFNR, volume-to-volume head motion, and number of motion spikes within the time series). All resulting z-statistic images were thresholded using a cluster-forming threshold of 2.3 and a corrected clustersignificance threshold of $p<0.05$. Although this threshold combined with FSL's FLAME 1 protects against false positives, we note that all of our results also survived permutation-based testing (Eklund et al., 2016). In these supplemental tests, statistical significance was assessed in a nonparametric fashion via FSL's randomise; this tool uses Monte Carlo permutation-based testing with 10000 permutations and $\alpha=0.05$, corrected for multiple comparisons across the whole brain (Nichols and Holmes, 2002; Winkler et al., 2014).

Brain images and activations are displayed using MRlcroGL (http://www.mccauslandcenter.sc.edu/mricrogl/; Rorden et al., 2007). All coordinates are reported in MNI space.

\section{Results}

\section{Previous stimulus category influences current behavior}

Social rewards, such as images of individuals or interactions with others, provide a useful tool for examining the effects of both social context and nonconsumable rewards on motivated behavior. However, relatively little is known about the effects of social and nonsocial rewards on future motivated behavior, and how the brain orchestrates future motivated action. To examine the effect of social rewards on subsequent motivated actions, we calculated a subsequent-RT effect by subtracting RTs following nonsocial trials from RTs following social trials. When averaging across value and delay trials, we found an overall effect of previous trial stimulus type on current trial RT: participants exhibited increased (slower) RTs subsequent to social trials $(\mathrm{M}=0.313 \mathrm{~s}, \mathrm{SD}=0.008 \mathrm{~s}$ ) compared to subsequent to nonsocial trials $(\mathrm{M}=0.306 \mathrm{~s}$, $\mathrm{SD}=0.008 \mathrm{~s} ; t_{(40)}=2.63, p=0.01, d=0.41$; Fig. $2 A$ ) This pattern replicated when examining hit trials only (social: $\mathrm{M}=0.317 \mathrm{~s}, \mathrm{SD}=0.009 \mathrm{~s}$; nonsocial: $\mathrm{M}=0.309$ $\left.\mathrm{s}, \mathrm{SD}=0.008 \mathrm{~s} ; t_{(40)}=2.63, p=0.01, d=0.41\right)$. Thus, participants were slower after performing a social trial compared to a nonsocial trial.

We next ran a GLM to control for properties of the previous and current trials (see Materials and Methods, Behavioral analysis). Across participants, current stimulus category was the strongest predictor of current RT $\left(t_{(40)}=\right.$ $-4.31, p<.0001, d=0.67)$, reflecting that participants were faster to respond during a social trial than during a nonsocial trial. The next strongest predictor was the previous trial's stimulus category $\left(t_{(40)}=2.46, p=0.01, d=\right.$ $0.38)$, reflecting that participants were slower to respond following a social trial than following a nonsocial trial. Neither previous trial's RT nor previous trial's reward attractiveness rating significantly predicted the current tri- 

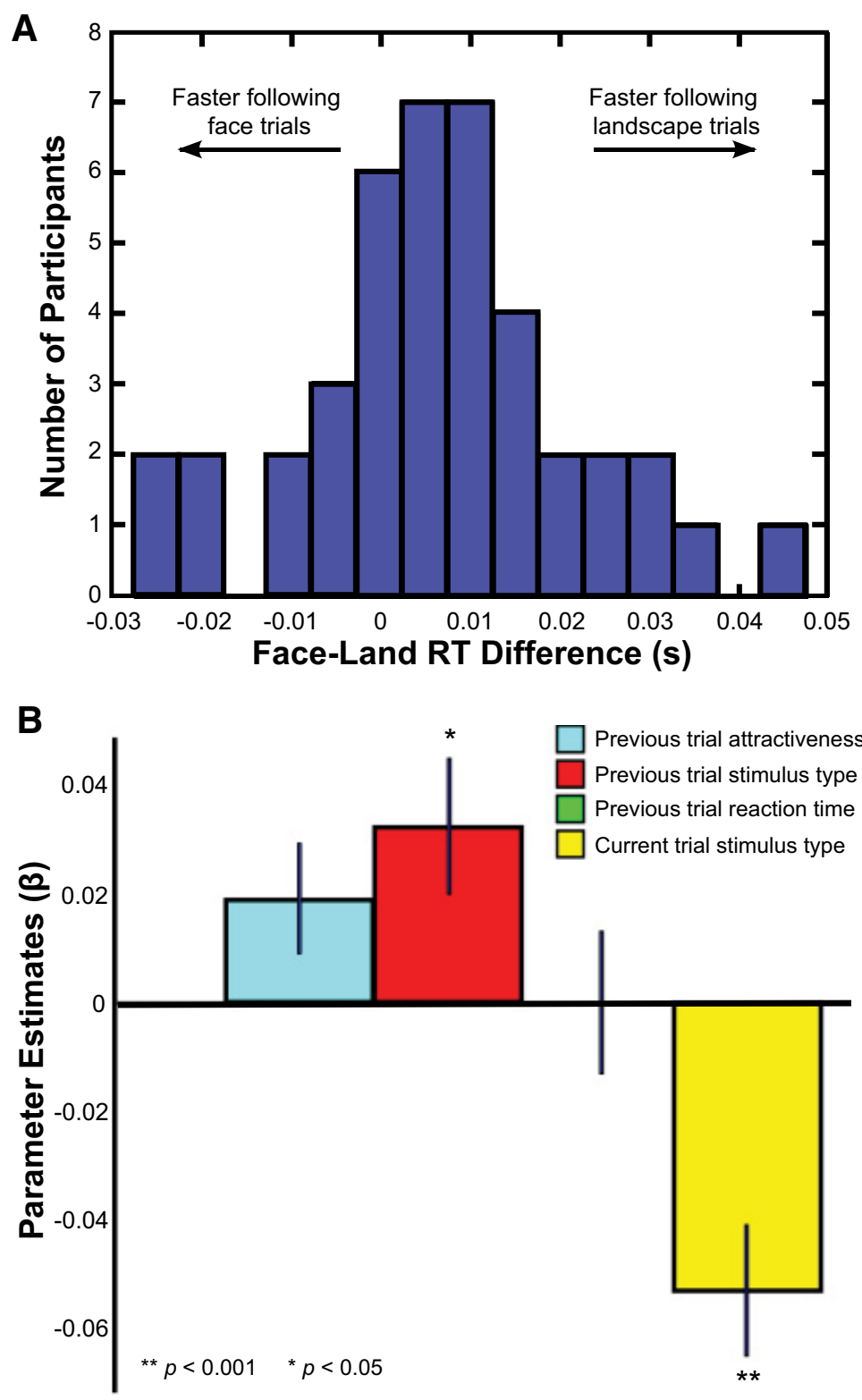

Figure 2. RTs are slower following face trials compared to landscape trials. A, Distribution of RT differences according to previous trial's stimulus type, calculated by subtracting RTs following nonsocial trials from RTs following social trials. RTs on trials following social rewards were greater than those on trials following nonsocial rewards, indicating an effect of previous reward stimulus type on subsequent behavior. $\boldsymbol{B}$, Average $\beta$ weights (with SEM plotted) across subjects from a behavioral regression predicting current RT. We regressed current trial RT on a model including the following regressors: stimulus category (social or nonsocial) on previous trial, RT on previous trial, attractiveness rating of the previous trial's reward, and stimulus category on current trial. Of these four regressors, current stimulus category most strongly predicted current RT; the negative $\beta$ weight indicates that participants are faster to respond during a social trial compared to during a nonsocial trial. The next most predictive regressor was previous trial's stimulus category; the positive $\beta$ weight indicates that participants were slower to respond following social trials compared to following nonsocial trials. Neither previous trial's RT nor previous trial's reward attractiveness significantly predicted the current trial's RT.

al's RT (Fig. 2B; Table 1). We note that the effect of previous stimulus type on current RT was still significant even after accounting for the large effect of current stimulus type, reflecting a distinct role of previous stimulus type unaccounted for by the other variables included in the GLM. These results indicate that of the measured properties of the previous trial, prior stimulus type had the strongest effect on current RT.

\section{Network coupling with FG tracks effect of stimulus category on subsequent behavior}

Our behavioral results indicated that current-trial stimulus type influences motivated behavior on the subsequent trial (which occurred $7 \mathrm{~s}$ or more later), such that participants were slower to respond following social trials compared to following nonsocial trials. However, it remains unclear how interactions in the brain during this 
Table 1. Behavioral regression results

\begin{tabular}{|c|c|c|c|}
\hline Regressor & Parameter estimate (SEM) & $t$ stat & $p$ value \\
\hline Current trial $(t)$ stimulus type & $-0.055(0.013)$ & -4.31 & $<0.0001$ \\
\hline Prior trial $(t-1)$ stimulus type & $0.033(0.013)$ & 2.46 & 0.01 \\
\hline Prior trial $(t-1)$ attractiveness & $0.019(0.011)$ & 1.74 & 0.09 \\
\hline Prior trial $(t-1) \mathrm{RT}$ & $<0.0001(0.013)$ & 0.005 & 0.99 \\
\hline
\end{tabular}

To examine the effects of other trial characteristics on current trial RT, we regressed current trial RT on a model including: stimulus category (social or nonsocial) on previous trial, RT on previous trial, attractiveness rating of the previous trial's reward, and stimulus category on current trial. Our analysis indicated that current stimulus type had the strongest effect on current RT; however, of all the characteristics from the prior trial, only prior stimulus type had a significant effect on current RT.

previous stimulus outcome affect the subsequent RT. To investigate this, we examined how effective connectivity with the ECN, a network implicated in cognitive control and goal-directed behavior, and the DMN, a network linked with task engagement orchestrate this change in future motivated behavior. We predicted that this subsequent-RT effect would be guided, in part, by changes in the coupling between these large-scale functional networks and domain-specific brain regions when viewing social images compared to when viewing nonsocial images. In particular, we predicted that the subsequent-RT effect would be driven by changes in the relative coupling of the $E C N$ and the DMN during the reward outcome phase of the previous trial. Our analysis pipeline allowed us to meet the criteria to claim that interactions between large-scale networks and specific brain regions are critical for behavior changes (see Introduction).

After running the ICA, we identified the DMN and ECN maps by running spatial correlations between the unthresholded maps from our ICA and the DMN and ECN maps from Smith et al. (2009). From our 25 components, we selected the maps that best matched the DMN $(R=$ 0.776; other components: $R_{\text {mean }}=-0.006, R_{\min }=$ $\left.-0.179, R_{\max }=0.124\right)$ and $\mathrm{ECN}(R=0.64$; other components: $R_{\text {mean }}=0.019, R_{\min }=-0.092, R_{\max }=0.296$ ) maps from Smith et al. (2009; Table 2). For ease of visualization, thresholded maps $(Z>4)$ are shown in Figure 3.

Following network identification, we ran a nPPI analysis that used participant-specific DMN and ECN time courses (estimated by the spatial regression analysis) as the physiologic regressors and presentation of social and nonsocial images as the psychological regressors. This nPPI identified regions that are influenced by the ECN and $\mathrm{DMN}$ in a task-dependent manner. We then tested whether these influences on cortex predicted the effect of prior stimulus type on current RT. Our nPPI analysis indicated that effective connectivity between the FG (peak: $x=38, y=-64, z=-20, p<0.0001$, voxel extent $=$ 383 ) and the ECN increased (compared to FG-DMN connectivity) when participants viewed social rewards (Fig. $4 A$ ), and that the magnitude of this increase tracked the slowing of RT on the subsequent trial (Fig. 4B). Strikingly, this peak voxel is consistent with the often functionallydefined FFA (McCarthy et al., 1997; Kanwisher and Yovel, 2006). Identification of the terms associated with this peak voxel using the meta-analytical tool Neurosynth (http:// neurosynth.org; Yarkoni et al., 2011) yielded "faces," "FFA," and "fusiform face" within its top four associations. These results suggest that functional networks associated with goal-directed and preparatory behavior can interact with focal brain regions to support task-relevant behavior.

To ensure that this connectivity was associated with the previous trial's outcome rather than the current trial's cue, we ran an additional nPPI to examine whole-brain connectivity during social cues, nonsocial cues, and nonsocial $>$ social cues. Notably, there were no regions exhibiting changes in connectivity with the ECN relative to the DMN in any of the three contrasts tested. To further investigate this, we used the FG region showing changes in ECN-DMN connectivity during the previous outcome phase as a mask during the current cue phase, and examined whether the ECN-DMN connectivity from this $\mathrm{ROI}$ tracked the effect of prior stimulus on current RT. Across the three contrasts tested (social, nonsocial, nonsocial > social), there was no correlation between the ROI connectivity estimates and the effect of previous stimulus

Table 2. Spatial correspondence with canonical networks

\begin{tabular}{lll}
\hline Canonical network & Independent component number & Spatial correlation $(r)$ \\
Visual 1 & IC10 & 0.82 \\
Visual 2 & IC01 & 0.66 \\
Visual 3 & IC01 & 0.45 \\
Default mode & IC06 & 0.78 \\
Cerebellar & IC18 & 0.32 \\
Sensorimotor & IC13 13 & 0.59 \\
Auditory & IC14 & 0.66 \\
Executive control & IC04 & 0.64 \\
R frontoparietal & IC03 & 0.64 \\
L frontoparietal & ICO & 0.77
\end{tabular}

To identify the DMN and ECN, we ran spatial correlations between canonical neural networks (Smith et al., 2009) and the 25 components from our ICA. The highest-correlating ICA component numbers for each network map and the correlation values are listed. 

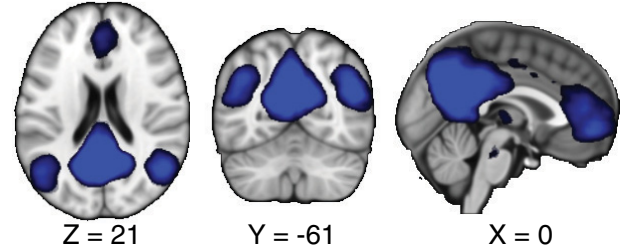

Default-Mode Network
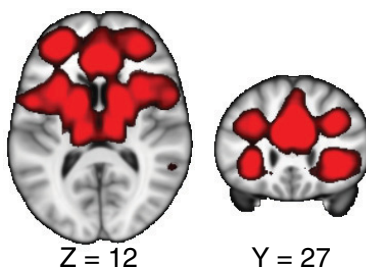

$\mathrm{Y}=27$

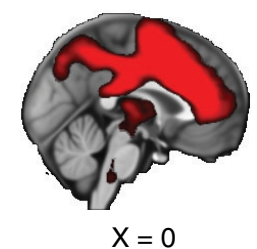

Executive Control Network

Figure 3. Networks identified from ICA. Using ICA across our task runs, we identified large-scale functional networks. This analysis produced 25 components. Our analyses focused on the components that best matched DMN (highest-correlating component illustrated on top) and the ECN (highest-correlating component illustrated on bottom) from Smith et al. (2009). For visualization purposes, maps are thresholded at $Z>4$.

type on current RT (social: $r=-0.12, p=0.45$; nonsocial: $r=0.01, p=0.94$; nonsocial > social: $r=0.23, p=0.15$ ). Collectively, these results support the claim that changes in subsequent $\mathrm{RT}$ are associated with differential network connectivity with the FG during the previous outcome phase, and cannot be attributed to other aspects of task performance.

No regions showed increased coupling with ECN (relative to $\mathrm{DMN}$ ) during nonsocial rewards that tracked the effect on subsequent RT, nor did any regions show increased coupling with DMN (relative to ECN) during social or nonsocial rewards that tracked the effect on subsequent RT. Additionally, there were no regions that exhibited changes in coupling with the DMN or ECN when comparing social and nonsocial rewards (e.g., DMNsocial > DMN-nonsocial; ECN-social > ECN-nonsocial; and the inverse contrasts) that tracked the effect on subsequent RT. Lastly, we ran a whole-brain GLM using the RT $\beta$ s as a covariate to examine whether any regions' activation tracked the effect of stimulus type on subsequent RT; we found that no regions tracked this effect using this traditional GLM. Thus, coupling between largescale networks and the FG that tracked the effect on subsequent RT was only observed during the viewing of social rewards.

\section{Discussion}

Recent neuroscience research has highlighted the relevance of large-scale functional networks to various aspects of behavior (Eichele et al., 2008; Kelly et al., 2008; Anticevic et al., 2010; Rosen et al., 2016). While many of these studies have linked network activation and connectivity to behavior, the contribution of these networks to motivated behaviors via focal cortical regions has been relatively understudied. For example, although previous work has found correlations between DMN and working memory (Sambataro et al., 2010; Piccoli et al., 2015) or
A
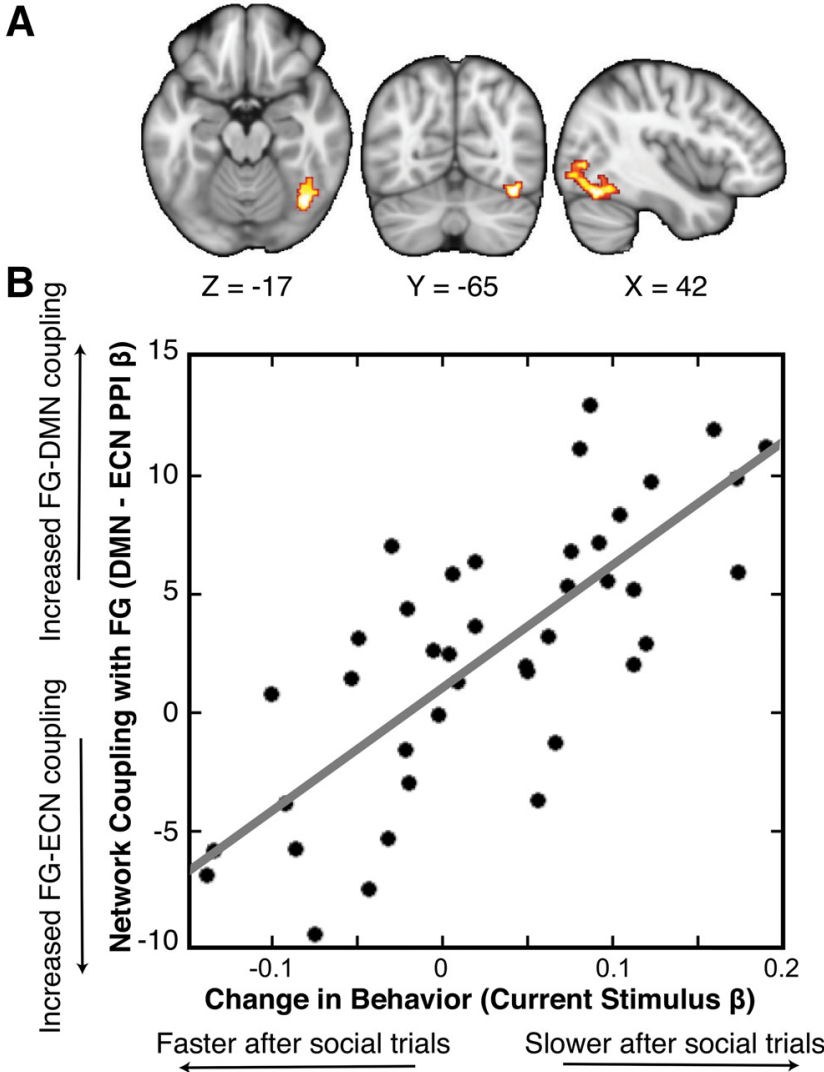

Figure 4. FG connectivity tracks effect of stimulus type on subsequent behavior. $\boldsymbol{A}, \mathrm{A} \mathrm{nPPI}$ indicated that an area in the $\mathrm{FG}$ exhibits heightened effective connectivity with ECN (compared to $\mathrm{DMN}$ ) during social reward outcomes; thresholded at $p<$ 0.05 . We note that this result also held with permutation-based testing (Eklund et al., 2016). B, Parameters estimates extracted from the FG connectivity track the effect of stimulus type on subsequent behavior (stimulus-type $\beta$ weights estimated from our behavioral GLM; see Materials and Methods, Behavioral analysis): as FG-DMN connectivity increases relative to FG-ECN connectivity, RTs are further slowed following social trials compared to following nonsocial trials.

sustained attention (Bonnelle et al., 2011; Gui et al., 2015), understanding how these distributed functional networks influence other cortical regions to shape behavior has remained a significant challenge. Here, we found that participants were slower in a RT task after having performed a trial for a social reward relative to a nonsocial reward (after accounting for the influence of the current trial cue type), reflecting a change in motivated behavior according to previous social stimulus type (Clithero et al., 2011). We then examined the neural mechanisms underlying this effect using nPPI analysis, an adaptation of generalized PPI analysis (i.e., effective connectivity) that examines effective connectivity with large-scale functional networks. This analysis pipeline allowed us to identify changes in coupling between large-scale networks and focal brain regions that predicted subsequent motivated behavior. Our results demonstrate that two goalrelevant networks, the $D M N$ and $E C N$, interact with $F G$ in a manner that predicts trial-to-trial adjustments in RT. 
Our analysis on our modified MID task identified that participants exhibit slower reaction times on trials subsequent to social outcomes, relative to trials subsequent to nonsocial outcomes. These results are consistent with a difference in motivational processes due to the social nature of the previous reward outcome, analogous to effects on motivation observed in similar tasks with nonsocial stimuli (Clithero et al., 2011). There are at least two potential explanations for this subsequent reaction time effect. The first hypothesis is that social images are more distracting than nonsocial images, and participants may still covertly attend to a prior social reward more so than they do a prior nonsocial reward. Previous research indicates that social images interfere with visual attention to a greater degree than nonsocial images (Ebitz et al., 2013); thus, it is possible that this differential interference may affect subsequent trials differently, as well. A second and related potential explanation is that participants' decreased motivation can be attributed to the satisfaction of receiving a motivating reward on the previous trial, much akin to results seen in "satisfaction of search" (SOS) research. In SOS research, participants performing visual search tasks tend to discontinue their search after finding an initial item, and either miss or display slower reaction times for subsequent items (Berbaum et al., 1990; Fleck et al., 2010). As applied to our MID task, a more motivating reward outcome (social images) may hinder the performance on the subsequent trial. Future research will be needed to explore the cognitive mechanisms driving the observed subsequent-RT effect and to determine if it is specific to social versus nonsocial stimuli, or may generalize to items that are more motivating versus less motivating.

Our findings expand on recent research examining the relevance of DMN and ECN activation to behavior, potentially in task-relevant contexts. Specifically, we found that increased ECN (relative to DMN) coupling with the FG is associated with enhanced subsequent task performance. These results are consistent with previous studies demonstrating different relationships between the DMN and ECN with task behavior: while ECN activation is often associated with heightened task performance and behavior (Dosenbach et al., 2007; Seeley et al., 2007), DMN activation is frequently linked to decrements in behavior and engagement in both humans (Weissman et al., 2006; Eichele et al., 2008) and nonhuman primates (Hayden et al., 2009, 2010; Heilbronner and Platt, 2013). Importantly, however, our findings extend these previous results by demonstrating direct coupling of these networks with a prototypical social-perception processing region in a task-dependent manner. Our results additionally highlight that the opposing effects of DMN and ECN are not limited to concurrent behavior, but also affect subsequent behavior with effects observed seven or more seconds later. These findings support the idea that large-scale networks interact with lower-level perceptual regions to contribute to motivational processes (Clithero et al., 2011) and shape later behavior.

Unlike previous studies examining large-scale networks (Seeley et al., 2007; Brewer et al., 2011; Ossandon et al.,
2011; Utevsky et al., 2014), our experiment demonstrates network coupling that directly shapes subsequent RT. While prior studies report associations between functional connectivity and behavior, estimates of functional connectivity solely report on correlations in activation (not coupling) between regions, which can be the result of various phenomena (Friston, 2011). Specifically, reported changes in functional connectivity can arise from numerous causes, including: changes in connectivity with another region, changes in observation noise (or signalto-noise ratios), and changes in the degree of neuronal fluctuations (for a review, see Friston, 2011). Thus, changes in functional connectivity may not reflect changes in coupling between cortical regions. In contrast to functional connectivity, we implemented an adaptation of a traditional PPI analysis to measure effective connectivity between functional networks and other cortical regions. PPI analyses measure whether a psychological context (e.g., outcome stimulus category) influences how one brain region or network (the "seed") contributes to another (the "target") by examining whether an interaction between the psychological context and the seed is identified in the target (Smith et al., 2016). Thus, a PPI analysis can eschew the potential confounds of functional connectivity analyses and reflects a change in neural coupling (e.g., effective connectivity). We note that our novel approach of generalized nPPI, applying generalized PPI analyses to large-scale networks, allows us to examine task-dependent contributions between networks and other cortical regions (Friston et al., 1997; Friston, 2011). In this way, our study extends prior work by demonstrating specific task-dependent coupling of the DMN/ECN.

Although recent meta-analytic work has demonstrated that PPI produces consistent and specific patterns of connectivity (Smith et al., 2016; Smith and Delgado, 2017), it is important to note that PPI results can be interpreted in two ways (Friston et al., 1997). First, our effects could reflect a context-specific modulation of effective connectivity. In this case, face presentations modulate the degree to which the DMN and ECN contribute to FG. Our results focus on the difference between DMN and ECN contributions to FG which seem to facilitate social motivation (Clithero et al., 2011). Alternatively, our effects could reflect a modulation of stimulus-specific responses. In this case, the DMN and ECN influence how FG responds to the presentation of the face; under this interpretation, our results suggest that the degree to which $D M N$ enhances face responses in $F G$ is greater than that of ECN. In either interpretation, the resulting effect on FG connectivity predicts behavior. A better understanding of the mechanisms and causal relationship underlying our results may be facilitated by other analytical approaches, such as dynamic causal modeling (DCM; Friston et al., 2003; Friston, 2011), although relatively less is understood regarding how the biophysical models implemented in DCM apply to distributed functional networks (Buxton et al., 1998; Friston et al., 2003). Additionally, other methodological approaches, such as transcranial magnetic stimulation (Fox et al., 2012; Luber and Lisanby, 2014; Mueller et al., 2014) or transcranial current stimulation 
(Keeser et al., 2011; Hampstead et al., 2014) may also better inform the specific interactions between FG and the ECN and DMN.

One potential caveat to note is that our task design included a cue to indicate the current trial's stimulus type in between the previous trial's outcome phase and the current trial's response phase. Because the current trial's stimulus type is the strongest predictor of the current trial's RT, there may be concerns over whether the neural coupling we observe can be attributed to the cue, rather than the previous trial's outcome. This concern is ameliorated through both the randomization of trial types (i.e., the identity of the outcome on the previous trial is uncorrelated with the trial type on the subsequent trial), as well as the run-level models implemented in our PPI analysis that included separate regressors for both the outcome phase (social and nonsocial outcomes, separately) and the cue phase (social and nonsocial cues, separately). Including these separate regressors for each phase allowed us to distinguish effects of the cue and outcome phases on connectivity. This concern is additionally mitigated by the analyses examining connectivity during the cue phase. A nPPI showed no changes in ECN-DMN connectivity during social cues, nonsocial cues, or social $>$ nonsocial cues when looking across the whole brain. A supplementary $\mathrm{ROI}$ analysis using the $\mathrm{FG}$ region indicated that FG connectivity with the ECN (relative to the DMN) during these three contrasts did not track the effect of the previous trial stimulus type on subsequent RT (as illustrated by the lack of significant correlations between the connectivity estimates and the effect of previous stimulus type on subsequent RT). However, future research may benefit from a modified task design in which there are no informative stimuli presented between a reward image and subsequent response.

As an additional caveat, we note that our paradigm and results leave room for interpretive challenges. Because participants exhibited faster RTs to view social images compared to nonsocial images, we cannot discern whether our nPPI results from the social-stimuli condition are due to the stimulus type itself (i.e., face images) or would generalize to other highly motivating stimuli. While prior work linking $F G$ to face processing supports the interpretation that our results are indeed due to that specific stimulus type (Kanwisher et al., 1997; McCarthy et al., 1997; Kanwisher and Yovel, 2006; Engell and McCarthy, 2014), future studies could compare withindomain images of varying attractiveness to other categories of motivating stimuli (e.g., money). Such an analysis would speak to whether these behavioral and neural results are unique to face image rewards, or are due to participant differences in subjective value (Bos et al., 2013; Smith et al., 2014a).

Our results demonstrate task-dependent contributions between the DMN and ECN and the FG that shape subsequent motivated behavior. These large-scale networks are known to be disrupted in a variety of psychopathologies marked by impairments in attention and reward processing, including autism spectrum disorder (Assaf et al., 2010; Young et al., 2015), obsessive compulsive disorder
(Stern et al., 2011, 2012), and major depressive disorder (Sheline et al., 2009; Grimm et al., 2011), and so an improved understanding of how they influence momentto-moment behavior could have clinical relevance and advance models of pathophysiology (Insel et al., 2010; Cuthbert and Insel, 2013). Thus, this study marks a significant step toward better understanding and treatment of disorders characterized by impaired social and reward processing.

\section{References}

Anticevic A, Repovs G, Shulman GL, Barch DM (2010) When less is more: TPJ and default network deactivation during encoding predicts working memory performance. Neuroimage 49:2638-2648. CrossRef

Assaf M, Jagannathan K, Calhoun VD, Miller L, Stevens MC, Sahl R, O'Boyle JG, Schultz RT, Pearlson GD (2010) Abnormal functional connectivity of default mode sub-networks in autism spectrum disorder patients. Neuroimage 53:247-256. CrossRef

Beckmann CF, Smith SM (2004) Probabilistic independent component analysis for functional magnetic resonance imaging. IEEE Trans Med Imaging 23:137-152. CrossRef

Beckmann CF, DeLuca M, Devlin JT, Smith SM (2005) Investigations into resting-state connectivity using independent component analysis. Philos Trans R Soc Lond B Biol Sci 360:1001-1013. CrossRef

Behrens TE, Sporns O (2012) Human connectomics. Curr Opin Neurobiol 22:144-153. CrossRef Medline

Berbaum KS, Franken EA, Dorfman DD, Rooholamini SA, Kathol MH, Barloon TJ, Behlke FM, Sato Y, Lu CH, el-Khoury GY (1990) Satisfaction of search in diagnostic radiology. Invest Radiol 25: 133-140. Medline

Biswal B, Zerrin Yetkin F, Haughton VM, Hyde JS (1995) Functional connectivity in the motor cortex of resting human brain using echo-planar mri. Magn Reson Med 34:537-541. CrossRef

Biswal BB, Mennes M, Zuo X-N, Gohel S, Kelly C, Smith SM, Beckmann CF, Adelstein JS, Buckner RL, Colcombe S, Dogonowski A-M, Ernst M, Fair D, Hampson M, Hoptman MJ, Hyde JS, Kiviniemi VJ, Kötter R, Li S-J, Lin C-P, et al. (2010) Toward discovery science of human brain function. Proc Natl Acad Sci USA 107:4734-4739. CrossRef

Bonnelle V, Leech R, Kinnunen KM, Ham TE, Beckmann CF, Boissezon XD, Greenwood RJ, Sharp DJ (2011) Default mode network connectivity predicts sustained attention deficits after traumatic brain injury. J Neurosci 31:13442-13451. CrossRef

Bos W, van den Talwar A, McClure SM (2013) Neural correlates of reinforcement learning and social preferences in competitive bidding. J Neurosci 33:2137-2146. CrossRef

Brainard DH (1997) The psychophysics toolbox. Spat Vis 10:433436. Medline

Brewer JA, Worhunsky PD, Gray JR, Tang Y-Y, Weber J, Kober H (2011) Meditation experience is associated with differences in default mode network activity and connectivity. Proc Natl Acad Sci USA 108:20254-20259. CrossRef

Buxton RB, Wong EC, Frank LR (1998) Dynamics of blood flow and oxygenation changes during brain activation: the balloon model. Magn Reson Med 39:855-864. CrossRef

Clithero JA, Reeck C, Carter RM, Smith DV, Huettel SA (2011) Nucleus accumbens mediates relative motivation for rewards in the absence of choice. Front Hum Neurosci 5:87. CrossRef

Cole DM, Smith SM, Beckmann CF (2010) Advances and pitfalls in the analysis and interpretation of resting-state FMRI data. Front Syst Neurosci 4:8. CrossRef Medline

Cowdrey FA, Filippini N, Park RJ, Smith SM, McCabe C (2014) Increased resting state functional connectivity in the default mode network in recovered anorexia nervosa. Hum Brain Mapp 35:483491. 
Cuthbert BN, Insel TR (2013) Toward the future of psychiatric diagnosis: the seven pillars of RDoC. BMC Med 11:126. CrossRef Medline

De Havas JA, Parimal S, Soon CS, Chee MWL (2012) Sleep deprivation reduces default mode network connectivity and anticorrelation during rest and task performance. Neuroimage 59: 1745-1751. CrossRef

Dosenbach NUF, Fair DA, Miezin FM, Cohen AL, Wenger KK, Dosenbach R. a T, Fox MD, Snyder AZ, Vincent JL, Raichle ME, Schlaggar BL, Petersen SE (2007) Distinct brain networks for adaptive and stable task control in humans. Proc Natl Acad Sci USA 104:11073-11078. CrossRef

Ebitz RB, Watson KK, Platt ML (2013) Oxytocin blunts social vigilance in the rhesus macaque. Proc Natl Acad Sci USA 110:1163011635. CrossRef Medline

Eichele T, Debener S, Calhoun VD, Specht K, Engel AK, Hugdahl K, von Cramon DY, Ullsperger M (2008) Prediction of human errors by maladaptive changes in event-related brain networks. Proc Natl Acad Sci USA 105:6173-6178. CrossRef

Eklund A, Nichols TE, Knutsson H (2016) Cluster failure: why fMRI inferences for spatial extent have inflated false-positive rates. Proc Natl Acad Sci USA 113:7900-7905. CrossRef

Engell AD, McCarthy G (2014) Face, eye, and body selective responses in fusiform gyrus and adjacent cortex: an intracranial EEG study. Front Hum Neurosci 8:642. CrossRef

Filippini N, Maclntosh BJ, Hough MG, Goodwin GM, Frisoni GB, Smith SM, Matthews PM, Beckmann CF, Mackay CE (2009) Distinct patterns of brain activity in young carriers of the APOE- $\epsilon 4$ allele. Proc Natl Acad Sci USA 106:7209-7214. CrossRef

Fleck MS, Samei E, Mitroff SR (2010) Generalized "satisfaction of search": adverse influences on dual-target search accuracy. J Exp Psychol 16:60-71. CrossRef

Fox MD, Halko MA, Eldaief MC, Pascual-Leone A (2012) Measuring and manipulating brain connectivity with resting state functional connectivity magnetic resonance imaging (fcMRI) and transcranial magnetic stimulation (TMS). Neuroimage 62:2232-2243. CrossRef

Friedman L, Glover GH (2006) Reducing interscanner variability of activation in a multicenter fMRI study: controlling for signal-tofluctuation-noise-ratio (SFNR) differences. Neuroimage 33:471481.

Friston KJ (2011) Functional and effective connectivity: a review. Brain Connect 1:13-36. CrossRef Medline

Friston KJ, Buechel C, Fink GR, Morris J, Rolls E, Dolan RJ (1997) Psychophysiological and modulatory interactions in neuroimaging. Neuroimage 6:218-229. CrossRef Medline

Friston KJ, Harrison L, Penny W (2003) Dynamic causal modelling. Neuroimage 19:1273-1302. CrossRef

Grimm S, Ernst J, Boesiger P, Schuepbach D, Boeker H, Northoff G (2011) Reduced negative BOLD responses in the default-mode network and increased self-focus in depression. World $\mathrm{J}$ Biol Psychiatry 12:627-637. CrossRef Medline

Gui D, Xu S, Zhu S, Fang Z, Spaeth AM, Xin Y, Feng T, Rao H (2015) Resting spontaneous activity in the default mode network predicts performance decline during prolonged attention workload. Neuroimage 120:323-330.

Gusnard DA, Raichle ME (2001) Searching for a baseline: functional imaging and the resting human brain. Nat Rev Neurosci 2:685694. CrossRef

Hampstead BM, Brown GS, Hartley JF (2014) Transcranial direct current stimulation modulates activation and effective connectivity during spatial navigation. Brain Stimulat 7:314-324. CrossRef

Hayden BY, Smith DV, Platt ML (2009) Electrophysiological correlates of default-mode processing in macaque posterior cingulate cortex. Proc Natl Acad Sci USA 106:5948-5953. CrossRef

Hayden BY, Smith DV, Platt ML (2010) Cognitive control signals in posterior cingulate cortex. Front Hum Neurosci 4:223. CrossRef

Heilbronner SR, Platt ML (2013) Causal evidence of performance monitoring by neurons in posterior cingulate cortex during learning. Neuron 80:1384-1391. CrossRef
Hyvärinen A (1999) Fast and robust fixed-point algorithms for independent component analysis. IEEE Trans Neural Netw 10:626634. CrossRef Medline

Insel T, Cuthbert B, Garvey M, Heinssen R, Pine DS, Quinn K, Sanislow C, Wang P (2010) Research domain criteria (RDoC): toward a new classification framework for research on mental disorders. Am J Psychiatry 167:748-751. CrossRef

Jenkinson M, Smith S (2001) A global optimisation method for robust affine registration of brain images. Med Image Anal 5:143-156. CrossRef

Kanwisher N, Yovel G (2006) The fusiform face area: a cortical region specialized for the perception of faces. Philos Trans R Soc Lond B Biol Sci 361:2109-2128. CrossRef

Kanwisher N, McDermott J, Chun MM (1997) The fusiform face area: a module in human extrastriate cortex specialized for face perception. J Neurosci 17:4302-4311.

Keeser D, Meindl T, Bor J, Palm U, Pogarell O, Mulert C, Brunelin J, Moller H-J, Reiser M, Padberg F (2011) Prefrontal transcranial direct current stimulation changes connectivity of resting-state networks during fMRI. J Neurosci 31:15284-15293. CrossRef

Kelly A, Uddin LQ, Biswal BB, Castellanos FX, Milham MP (2008) Competition between functional brain networks mediates behavioral variability. Neuroimage 39:527-537. CrossRef

Knutson B, Westdorp A, Kaiser E, Hommer D (2000) FMRI visualization of brain activity during a monetary incentive delay task. Neuroimage 12:20-27. CrossRef

Knutson B, Adams CM, Fong GW, Hommer D (2001) Anticipation of increasing monetary reward selectively recruits nucleus accumbens. J Neurosci 21:RC159.

Kucyi A, Moayedi M, Weissman-Fogel I, Goldberg MB, Freeman BV, Tenenbaum HC, Davis KD (2014) Enhanced medial prefrontaldefault mode network functional connectivity in chronic pain and its association with pain rumination. J Neurosci 34:3969-3975. CrossRef

Leech R, Kamourieh S, Beckmann CF, Sharp DJ (2011) Fractionating the default mode network: distinct contributions of the ventral and dorsal posterior cingulate cortex to cognitive control. J Neurosci 31:3217-3224. CrossRef

Luber B, Lisanby SH (2014) Enhancement of human cognitive performance using transcranial magnetic stimulation (TMS). Neuroimage 85:961-970. CrossRef

McCarthy G, Puce A, Gore JC, Allison T (1997) Face-specific processing in the human fusiform gyrus. J Cogn Neurosci 9:605-610. CrossRef

McLaren DG, Ries ML, Xu G, Johnson SC (2012) A generalized form of context-dependent psychophysiological interactions (gPPI): a comparison to standard approaches. Neuroimage 61:1277-1286. CrossRef

Mueller JK, Grigsby EM, Prevosto V, Petraglia lii FW, Rao H, Deng Z-D, Peterchev AV, Sommer MA, Egner T, Platt ML, Grill WM (2014) Simultaneous transcranial magnetic stimulation and singleneuron recording in alert non-human primates. Nat Neurosci 17: 1130-1136.

Nichols TE, Holmes AP (2002) Nonparametric permutation tests for functional neuroimaging: a primer with examples. Hum Brain Mapp 15:1-25. CrossRef

O'Reilly JX, Woolrich MW, Behrens TE, Smith SM, Johansen-Berg H (2012) Tools of the trade: psychophysiological interactions and functional connectivity. Soc Cogn Affect. Neurosci 7:604-609.

Ossandon T, Jerbi K, Vidal JR, Bayle DJ, Henaff M-A, Jung J, Minotti L, Bertrand O, Kahane P, Lachaux J-P (2011) Transient suppression of broadband gamma power in the default-mode network is correlated with task complexity and subject performance. J Neurosci 31:14521-14530. CrossRef

Piccoli T, Valente G, Linden DEJ, Re M, Esposito F, Sack AT, Salle FD (2015) The default mode network and the working memory network are not anti-correlated during all phases of a working memory task. PLoS One 10:e0123354. CrossRef Medline 
Ray KL, McKay DR, Fox PM, Riedel MC, Uecker AM, Beckmann CF, Smith SM, Fox PT, Laird AR (2013) ICA model order selection of task co-activation networks. Front Neurosci 7:237. CrossRef

Rorden C, Karnath H-O, Bonilha L (2007) Improving lesion-symptom mapping. J Cogn Neurosci 19:1081-1088. CrossRef Medline

Rosen ML, Stern CE, Michalka SW, Devaney KJ, Somers DC (2016) Cognitive control network contributions to memory-guided visual attention. Cereb Cortex 26:2059-2073.

Sambataro F, Murty VP, Callicott JH, Tan H-Y, Das S, Weinberger DR, Mattay VS (2010) Age-related alterations in default mode network: impact on working memory performance. Neurobiol Aging 31:839-852. CrossRef

Seeley WW, Menon V, Schatzberg AF, Keller J, Glover GH, Kenna H, Reiss AL, Greicius MD (2007) Dissociable intrinsic connectivity networks for salience processing and executive control. J Neurosci 27:2349-2356. CrossRef

Shannon BJ, Raichle ME, Snyder AZ, Fair DA, Mills KL, Zhang D, Bache K, Calhoun VD, Nigg JT, Nagel BJ, Stevens AA, Kiehl KA (2011) Premotor functional connectivity predicts impulsivity in juvenile offenders. Proc Natl Acad Sci USA 108:11241-11245. CrossRef

Sheline YI, Barch DM, Price JL, Rundle MM, Vaishnavi SN, Snyder AZ, Mintun MA, Wang S, Coalson RS, Raichle ME (2009) The default mode network and self-referential processes in depression. Proc Natl Acad Sci USA 106:1942-1947. CrossRef Medline

Sladky R, Friston KJ, Tröstl J, Cunnington R, Moser E, Windischberger C (2011) Slice-timing effects and their correction in functional MRI. Neuroimage 58:588-594. CrossRef

Smith DV, Delgado MR (2017) Meta-analysis of psychophysiological interactions: revisiting cluster-level thresholding and sample sizes. Hum Brain Mapp 38:588-591.

Smith DV, Hayden BY, Truong T-K, Song AW, Platt ML, Huettel SA (2010) Distinct value signals in anterior and posterior ventromedial prefrontal cortex. J Neurosci 30:2490-2495. CrossRef

Smith DV, Clithero JA, Boltuck SE, Huettel SA (2014a) Functional connectivity with ventromedial prefrontal cortex reflects subjective value for social rewards. Soc Cogn Affect Neurosci 9:2017-2025. CrossRef

Smith DV, Utevsky AV, Bland AR, Clement N, Clithero JA, Harsch AEW, McKell Carter R, Huettel SA (2014b) Characterizing individual differences in functional connectivity using dual-regression and seed-based approaches. Neuroimage 95:1-12. CrossRef

Smith DV, Gseir M, Speer ME, Delgado MR (2016) Toward a cumulative science of functional integration: a meta-analysis of psychophysiological interactions. Hum Brain Mapp 37:2904-2917.

Smith SM (2002) Fast robust automated brain extraction. Hum Brain Mapp 17:143-155. CrossRef
Smith SM, Jenkinson M, Woolrich MW, Beckmann CF, Behrens TE, Johansen-Berg H, Bannister PR, De Luca M, Drobnjak I, Flitney DE, Niazy RK, Saunders J, Vickers J, Zhang Y, De Stefano N, Brady JM, Matthews PM (2004) Advances in functional and structural MR image analysis and implementation as FSL. Neuroimage 23 [Suppl 1]:S208-S219. CrossRef

Smith SM, Fox PT, Miller KL, Glahn DC, Fox PM, Mackay CE, Filippini N, Watkins KE, Toro R, Laird AR, Beckmann CF (2009) Correspondence of the brain's functional architecture during activation and rest. Proc Natl Acad Sci USA 106:13040-13045. CrossRef

Smith SM, Beckmann CF, Andersson J, Auerbach EJ, Bijsterbosch J, Douaud G, Duff E, Feinberg DA, Griffanti L, Harms MP, Kelly M, Laumann T, Miller KL, Moeller S, Petersen S, Power J, SalimiKhorshidi G, Snyder AZ, Vu AT, Woolrich MW, et al. (2013) Resting-state $\mathrm{fMRI}$ in the Human Connectome Project. Neuroimage 80:144-168. CrossRef

Stern ER, Fitzgerald KD, Welsh RC, Abelson JL, Taylor SF (2012) Resting-state functional connectivity between fronto-parietal and default mode networks in obsessive-compulsive disorder. PLoS One 7:e36356. CrossRef

Stern ER, Welsh RC, Fitzgerald KD, Gehring WJ, Lister JJ, Himle JA, Abelson JL, Taylor SF (2011) Hyperactive error responses and altered connectivity in ventromedial and frontoinsular cortices in obsessive-compulsive disorder. Biol Psychiatry 69:583-591. CrossRef

Utevsky AV, Smith DV, Huettel SA (2014) Precuneus is a functional core of the default-mode network. J Neurosci 34:932-940. CrossRef Medline

Weissman DH, Roberts KC, Visscher KM, Woldorff MG (2006) The neural bases of momentary lapses in attention. Nat Neurosci 9:971-978. CrossRef Medline

Winkler AM, Ridgway GR, Webster MA, Smith SM, Nichols TE (2014) Permutation inference for the general linear model. Neuroimage 92:381-397. CrossRef Medline

Woolrich MW, Jbabdi S, Patenaude B, Chappell M, Makni S, Behrens TE, Beckmann CF, Jenkinson M, Smith SM (2009) Bayesian analysis of neuroimaging data in FSL. Neuroimage 45:S173-S186. CrossRef Medline

Yarkoni T, Poldrack RA, Nichols TE, Van Essen DC, Wager TD (2011) Large-scale automated synthesis of human functional neuroimaging data. Nat Methods 8:665-670. CrossRef

Young JS, Smith DV, Coutlee CG, Huettel SA (2015) Synchrony between sensory and cognitive networks is associated with subclinical variation in autistic traits. Front Hum Neurosci 9:146. 